Draft Version DeCEMber 9, 2019

Typeset using LATEX preprint2 style in AASTeX61

\title{
NO SIGNIFICANT EVOLUTION OF RELATIONS BETWEEN BLACK HOLE MASS AND GALAXY TOTAL STELLAR MASS UP TO $Z \sim 2.5$
}

\author{
Hyewon Suh, ${ }^{1, *}$ Francesca Civano, ${ }^{2}$ Benny Trakhtenbrot,${ }^{3}$ Francesco Shankar,${ }^{4}$ \\ Günther Hasinger,${ }^{5}$ David B. Sanders, ${ }^{6}$ And Viola Allevato ${ }^{7}$ \\ ${ }^{1}$ Subaru Telescope, National Astronomical Observatory of Japan (NAOJ), 650 North A'ohoku place, Hilo, HI 96720, \\ USA \\ ${ }^{2}$ Harvard-Smithsonian Center for Astrophysics, Cambridge, MA, 02138, USA \\ ${ }^{3}$ School of Physics and Astronomy, Tel Aviv University, Tel Aviv 69978, Israel \\ ${ }^{4}$ Department of Physics and Astronomy, University of Southampton, Highfield SO17 1BJ, UK \\ ${ }^{5}$ European Space Astronomy Centre (ESA/ESAC), Director of Science, E-28691 Villanueva de la Cañada, Madrid, \\ Spain \\ ${ }^{6}$ Institute for Astronomy, University of Hawaii, 2680 Woodlawn Drive, Honolulu, HI 96822, USA \\ ${ }^{7}$ Scuola Normale Superiore, Piazza dei Cavalieri 7, I-56126 Pisa, Italy
}

\section{ABSTRACT}

We investigate the cosmic evolution of the ratio between black hole mass $\left(M_{\mathrm{BH}}\right)$ and host galaxy total stellar mass $\left(M_{\text {stellar }}\right)$ out to $z \sim 2.5$ for a sample of 100 X-ray-selected moderate-luminosity, broad-line active galactic nuclei (AGNs) in the Chandra-COSMOS Legacy Survey. By taking advantage of the deep multi-wavelength photometry and spectroscopy in the COSMOS field, we measure in a uniform way the galaxy total stellar mass using a SED decomposition technique and the black hole mass based on broad emission line measurements and single-epoch virial estimates. Our sample of AGN host galaxies has total stellar masses of $10^{10-12} M_{\odot}$, and black hole masses of $10^{7.0-9.5} M_{\odot}$. Combining our sample with the relatively bright AGN samples from the literature, we find no significant evolution of the $M_{\mathrm{BH}}-M_{\text {stellar }}$ relation with black hole-to-host total stellar mass ratio of $M_{\mathrm{BH}} / M_{\text {stellar }} \sim 0.3 \%$ at all redshifts probed. We conclude that the average black hole-to-host stellar mass ratio appears to be consistent with the local value within the uncertainties, suggesting a lack of evolution of the $M_{\mathrm{BH}}-M_{\text {stellar }}$ relation up to $z \sim 2.5$.

Keywords: galaxies: evolution - galaxies: active - galaxies: nuclei

suh@naoj.org

* Subaru Fellow 


\section{INTRODUCTION}

The local universe provides clear evidence that the growth of supermassive black holes (SMBHs) is closely connected with galaxy evolution, as revealed by well-known tight correlations between the black hole $(\mathrm{BH})$ mass and their host bulge properties (i.e., $M_{\mathrm{BH}}-$ $M_{\text {bulge }}, M_{\mathrm{BH}}-\sigma$ relations; Magorrian et al. 1998; Ferrarese \& Merritt 2000; Gebhardt et al. 2000; Häring \& Rix 2004; Gültekin et al. 2009; Sani et al. 2011; McConnell \& Ma 2013; Kormendy 2013; Shankar et al. 2016). Several studies have reported that the mass ratio between $\mathrm{BH}$ and host bulge is $M_{\mathrm{BH}} / M_{\text {bulge }} \sim 10^{-3}$ with intrinsic dispersion of $\sim 0.3$ dex (e.g., Marconi \& Hunt 2003; Häring \& Rix 2004; Sani et al. 2011). Kormendy \& Ho (2013) argued for larger values of $M_{\mathrm{BH}} / M_{\text {bulge }} \sim 10^{-2.3}$, mainly because of their revision of increased $\mathrm{BH}$ masses. Many theoretical models have proposed active galactic nucleus (AGN) feedback to explain these physical connection (e.g., Silk \& Rees 1998; Di Matteo et al. 2005; Hopkins et al. 2006), and yet, how SMBHs and their host galaxies evolve onto these local scaling relations through cosmic time remains unclear.

While relations between $\mathrm{BH}$ mass and bulge properties in quiescent bulge-dominant galaxies show the tightest correlation, for high redshift studies $(z>1)$ it is difficult to estimate the bulge mass due to the lack of spatial resolution and sensitivity, and thus, the total stellar mass is used instead, estimated by assuming a mass-to-light ratio or a spectral energy distribution (SED) fitting (e.g., Merloni et al. 2010; Cisternas et al. 2011; Bongiorno et al. 2014). Measuring the BH mass is also challenging beyond the local universe, and the virial method is often used to estimate the $\mathrm{BH}$ mass for galaxies hosting broadline AGN (e.g., Kaspi et al. 2000; Vestergaard 2002; Woo \& Urry 2002; McLure \& Jarvis 2002; McLure \& Dunlop 2004; Greene \& Ho 2005;
Kollmeier et al. 2006; Vestergaard \& Peterson 2006; Shen et al. 2008, 2011; Trakhtenbrot \& Netzer 2012).

Reines and Volonteri (2015) have quantified the relationship between $\mathrm{BH}$ mass and total stellar mass for nearby galaxies to facilitate work at higher redshifts, including both galaxies with quiescent and active BHs. They found that local AGN host galaxies tend to fall below the canonical BH-to-bulge mass relations deffined by inactive early-type galaxies at a given total stellar mass, by more than an order of magnitude, with $M_{\mathrm{BH}} / M_{\text {stellar }} \sim 10^{-4.6}$. Shankar et al. (2016, see also Bernardi et al. 2007; Shankar et al. 2017) used detailed Monte Carlo simulations to put forward evidence for a possible bias in the local $M_{\mathrm{BH}}-M_{\text {stellar }}$ relation of inactive $\mathrm{BHs}$ with dynamicallymeasured masses. They showed that, especially in early-type galaxies, the necessary requirement of resolving the gravitational sphere of influence of the central $\mathrm{BH}$ for reliable dynamical mass measurements can, by itself, bias upwards the $M_{\mathrm{BH}}-M_{\text {stellar }}$ relation, and proposed an intrinsic scaling $M_{\mathrm{BH}}-M_{\text {stellar }}$ relation, in the hypothesis that the mass of the $\mathrm{BH}$ is predominantly dependent on velocity dispersion. Shankar et al. (2019) further showed that local AGNs, which do not suffer from this selection effect, tend instead to naturally sit around the intrinsic $M_{\mathrm{BH}}-M_{\text {stellar }}$ relation proposed by Shankar et al. (2016, see also Reines and Volonteri 2015).

Many observational studies have found that SMBHs beyond the local universe are overmassive at a given host stellar mass compared with that at the present time, suggesting that BHs were able to grow more efficiently than their host galaxies (e.g., Peng et al. 2006; Treu et al. 2007; Woo et al. 2008; Merloni et al. 2010; Decarli et al. 2010; Trakhtenbrot \& Netzer 2010; Bennert et al. 2011; Caplar et al. 2015; Park et al. 2015; Trakhtenbrot et al. 2015; 
Caplar et al. 2018). Peng et al. (2006) suggested that the $M_{\mathrm{BH}} / M_{\text {stellar }}$ ratio is by a factor of $>\sim 4$ times larger at $z>1.7$ than today. Decarli et al. (2010) also claimed that the BHto-host mass ratios significantly increase by a factor of $\sim 7$ at $z=3$ from a sample of 96 quasars. Merloni et al. (2010) reported that the average $\mathrm{BH}$ to host galaxy mass ratio appears to evolve positively with redshift, with $M_{\mathrm{BH}} / M_{\text {stellar }} \propto(1+z)^{0.68}$.

On the other hand, some others found that the relationship between SMBHs and their host masses matches the correlation that we observe today, suggesting no evolution in the scaling relation within the uncertainties (e.g., Shields et al. 2003; Salviander et al. 2007; Shen et al. 2008; Shankar et al. 2009; Cisternas et al. 2011; Schramm \& Silverman 2013; Salviander et al. 2015; Shen et al. 2015; Sun et al. 2015). Jahnke et al. (2009) suggested no evolution in the $M_{\mathrm{BH}}-M_{\text {stellar }}$ relation using 10 of the targets in Merloni et al. (2010) sample when they independently derive host galaxy properties using HST observations. They found some evidence of substantial disk components from their HST imaging, suggesting that if the objects were purely bulge-dominated, the $M_{\mathrm{BH}}-M_{\text {stellar }}$ relation has not evolved, or at least not as rapidly as the relations between $\mathrm{BH}$ mass and spheroid properties since $z \sim 2$.

However, selection biases could affect the interpretation of the results on these relations at high redshift (e.g., Lauer et al. 2007; Treu et al. 2007; Schulze \& Wisotzki 2014; Shen et al. 2015). Lauer et al. (2007) pointed out that high redshift samples are generally selected by nuclear activity (i.e., AGN luminosity), and therefore, biased toward the most luminous AGNs. Such luminous systems are intrinsically rare and do not represent the typical AGN population (Richards et al. 2006; Ross et al. 2013; Aird et al. 2015). Due to this bias, luminous AGNs are more likely to be found in less mas- sive galaxies at higher redshift, resulting in an apparent evolution of the $M_{\mathrm{BH}}-M_{\text {stellar rela- }}$ tion.

In this paper, we make use of a large, deep/uniform X-ray depth and the extensive multi-wavelength photometric and spectroscopic data of Chandra-COSMOS Legacy Survey (Civano et al. 2016) to investigate the cosmic evolution of the relationship between $\mathrm{BH}$ mass and galaxy total stellar mass up to $z \sim 2.5$. The redshift range of $1<z<3$ is an essential cosmic period for studying the link between $\mathrm{BH}$ growth and galaxy evolution, which corresponds to the peak epoch of star formation and AGN activity. Our sample of X-ray-selected AGNs with lower luminosities $\left(L_{\text {bol }} \sim 10^{44-46} \mathrm{erg} \mathrm{s}^{-1}\right)$, together with the deep high-quality spectroscopy, contains a much more representative population of SMBHs and host galaxies, and is therefore less susceptible to the selection biases induced by flux limit effects, as compared to previous, mostly optical, samples. The BH mass estimate is based on the single-epoch virial method using the Keck/DEIMOS optical and Subaru/FMOS NIR spectroscopy, and the stellar mass is measured via a multi-component spectral energy distribution (SED) fitting. These measurements then allow us to investigate the evolution of $\mathrm{BH}$ galaxy scaling relations up to $z \sim 2.5$, for the deepest/largest data set adopted so far in this kind of studies.

Throughout this work, we adopt a standard $\Lambda \mathrm{CDM}$ cosmology with $\Omega_{m}=0.3, \Omega_{\Lambda}=0.7$, and $H_{0}=70 \mathrm{~km} \mathrm{~s}^{-1} \mathrm{Mpc}^{-1}$.

\section{BROAD-LINE AGN SAMPLE AND DATA}

We construct our sample of broad-line AGNs from the Chandra-COSMOS Legacy Survey (CCLS; Civano et al. 2016) with a multiwavelength data set (Marchesi et al. 2016; Laigle et al. 2016), which contains a total of $4016 \mathrm{X}$-ray sources down to a flux limit of $2.2 \times$ $10^{-16}, 1.5 \times 10^{-15}$, and $8.9 \times 10^{-16} \mathrm{erg} \mathrm{cm}^{-2} \mathrm{~s}^{-1}$ 

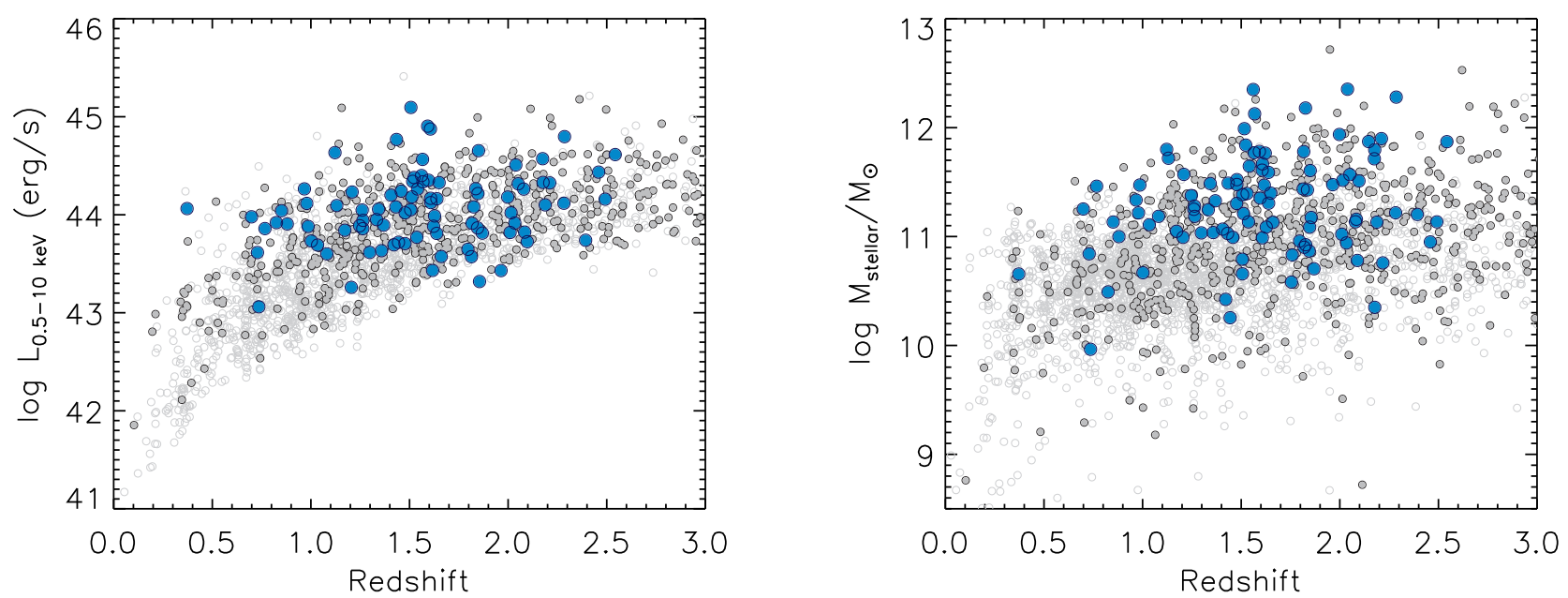

Figure 1. Absorption-corrected $0.5-10 \mathrm{keV}$ X-ray luminosity (left) and stellar mass (right) vs. redshift (spectroscopic or photometric) distribution for all X-ray sources in the CCLS. The sources classified as Type 1 (optically unobscured and/or broad-line) AGNs are denoted by filled gray circles (see Marchesi et al. 2016; Suh et al. 2019 for details). Our final sample of broad-line AGNs is shown as filled blue circles.

at 20 per cent completeness in the $0.5-2$ $\mathrm{keV}, 2-10 \mathrm{keV}$, and $0.5-10 \mathrm{keV}$ bands. Recently, Hasinger et al. (2018) presented the first comprehensive spectroscopic observations with Keck/DEIMOS (Faber et al. 2003) over a large area of $\sim 2.2 \mathrm{deg}^{2}$ in the COSMOS field (DEIMOS 10K spectroscopic survey). Compared with the previous spectroscopic surveys in the COSMOS field, such as the zCOSMOS bright spectroscopic catalog (Lilly et al. 2007) containing 10,644 spectra for a sample of galaxies with $I_{\mathrm{AB}}<22.5 \mathrm{mag}$, the DEIMOS $10 \mathrm{~K}$ spectroscopic catalog provides spectroscopy with a spectral resolution $R \sim 2000-2700$ for a sample of 10,718 objects with $I_{\mathrm{AB}}<23.5-25$ mag, including the newly-detected deep Chandra X-ray sources selected from CCLS. With the deeper magnitude limits, we expect to obtain a lower limit to the $\mathrm{BH}$ masses of $\log M_{\mathrm{BH}} \sim 7.04 M_{\odot}$ at $z=2$ using the methods detailed in Section 4. Furthermore, the FMOSCOSMOS spectroscopic survey was conducted with the Subaru/FMOS (Kimura et al. 2010) NIR high-resolution spectrographs $(R \sim 2200)$, described in detail in Silverman et al. (2015) (see also Kashino et al. 2013; Schulze et al. 2018). Our sample, therefore, represents the most typical AGN population covering lower luminosities, compared to previous studies at $z>1$.

We select our sample of broad-line AGNs, for which one or more broad emission lines with a full width at half-maximum (FWHM) larger than $2000 \mathrm{~km} \mathrm{~s}^{-1}$ have been identified by analyzing optical spectra of 1078 sources with DEIMOS, and additionally NIR spectra of 589 sources with FMOS. We detect $21 \mathrm{H} \alpha, 5 \mathrm{H} \beta$, and $74 \mathrm{Mg}$ II broad emission lines, for which having $\mathrm{S} / \mathrm{N}$ greater than 10 per pixel, in the optical/NIR spectra, respectively. We finally obtain 100 broad-line AGNs, covering the redshift range $z=0-2.5$ (Table 1 ).

In Figure 1, we show the distribution of the absorption-corrected 0.5-10 keV X-ray luminosity (left) and total stellar mass (right; Suh et al. 2019) as a function of redshift for all the CCLS sources. The final sample of our broad-line AGNs covers the full region of the X-ray luminosity $\left(L_{0.5-10 \mathrm{keV}} \sim 10^{43-45} \mathrm{erg} \mathrm{s}^{-1}\right)$ and stellar mass $\left(M_{\text {stellar }} \sim 10^{10-12} M_{\odot}\right)$ as the overall 
CCLS Type 1 AGN sample spanning about 2 dex at $1<z<2$.

\section{HOST STELLAR MASS}

The total stellar mass of our sample of broad-line AGN host galaxies is adopted from Suh et al. (2019) by performing a multi-component SED fitting from far-IR $(500 \mu \mathrm{m})$ to near-UV (2300A). Suh et al. (2019) decomposed the SED using an AGN accretion disk emission model (i.e., big blue bump) from Richards et al. (2006), a dust torus model from Silva et al. (2004), a starburst template from Chary \& Elbaz (2001) and Dale \& Helou (2002), and a galaxy stellar population model of Bruzual \& Charlot (2003). For the galaxy model template, they used the Chabrier (2003) initial mass function (IMF) and an exponentially decaying star formation histories with characteristic times ranging from $\tau=0.1$ to 30 Gyr and constant star formation. The law of Prevot et al. (1984) for an AGN accretion disk template and the law of Calzetti et al. (2000) for a set of galaxy templates are used to take into account the reddening effect. Four dust torus templates are used depending on the amount of nuclear obscuration.

Due to the degeneracy between the AGN accretion disk and the galaxy emission in the UVoptical wavelengths of SED fitting, we further require that the $\mathrm{AGN}$ emission dominates the galaxy light in the UV bands ( $>50 \%$ of total) by the fact that the UV/optical emission should come from the AGN accretion disk for Type 1 AGNs. Therefore, the stellar mass for the bestfit with this constraint could be biased toward the upper limit (see Suh et al. 2019). The typical uncertainties for the stellar mass are +0.19 dex and -0.36 dex toward higher and lower masses, respectively. A full detailed description is presented in Suh et al. $(2017,2019)$.

\section{BH MASS AND ACCRETION RATE}

The virial $\mathrm{BH}$ mass can be estimated using the broad-line width and the continuum/line luminosity from the single-epoch, rest-frame UV/optical spectra as a proxy for the characteristic velocity and the size of the broadline region (BLR; e.g., Vestergaard 2002; McLure \& Jarvis 2002; McLure \& Dunlop 2004; Greene \& Ho 2005; Vestergaard \& Peterson 2006; Shen et al. 2011; Trakhtenbrot \& Netzer 2012; Mejía-Restrepo et al. 2016). We measure the spectral complexes of broad $\mathrm{H} \alpha, \mathrm{H} \beta$, and $\mathrm{Mg}$ II emission lines present in optical and NIR spectra, depending on the redshift, to derive the virial BH mass of broad-line AGNs. We use the mpfit routine to fit the emission lines, which adopts a Levenberg-Marquardt least-squares minimization algorithm to derive the best-fit parameters and a measure of the goodness of the overall fit. The full details of the fit procedure are described in detail in Suh et al. (2015).

In short, we apply the absolute flux calibration by matching the deep ground-based photometry available in the COSMOS field. For $\mathrm{H} \alpha$ we fit and subtract a power-law continuum, $f_{\lambda} \propto \lambda^{-\alpha}$, from the spectra, and fit the narrow emission lines of $[\mathrm{N}$ II $] \lambda 6548,6583 \AA$ with a fixed ratio of 2.96 , and $\mathrm{H} \alpha \lambda 6563 \AA$. The broad $\mathrm{H} \alpha$ line is fit by one or two broad Gaussian components. In the case of the $\mathrm{H} \beta$ and $\mathrm{Mg}$ II lines, we simultaneously fit the combination of the power-law continuum and the FeII emission components (e.g., Vestergaard \& Wilkes 2001; Matsuoka et al. 2007; Harris et al. 2013), for which we adopt an empirical template from Vestergaard \& Wilkes (2001) convolved with Gaussian profiles of various widths. Then we subtract the best-fit power-law continuum and the FeII emission complex from the spectra. For $\mathrm{H} \beta$, the narrow line model is composed of $\mathrm{H} \beta \lambda 4861 \AA$ and $[\mathrm{O}$ III $] \lambda 4959,5007 \AA$ with a fixed ratio of 2.98. We fit the broad $\mathrm{H} \beta$ line with one or two broad Gaussian components. For MgII, 

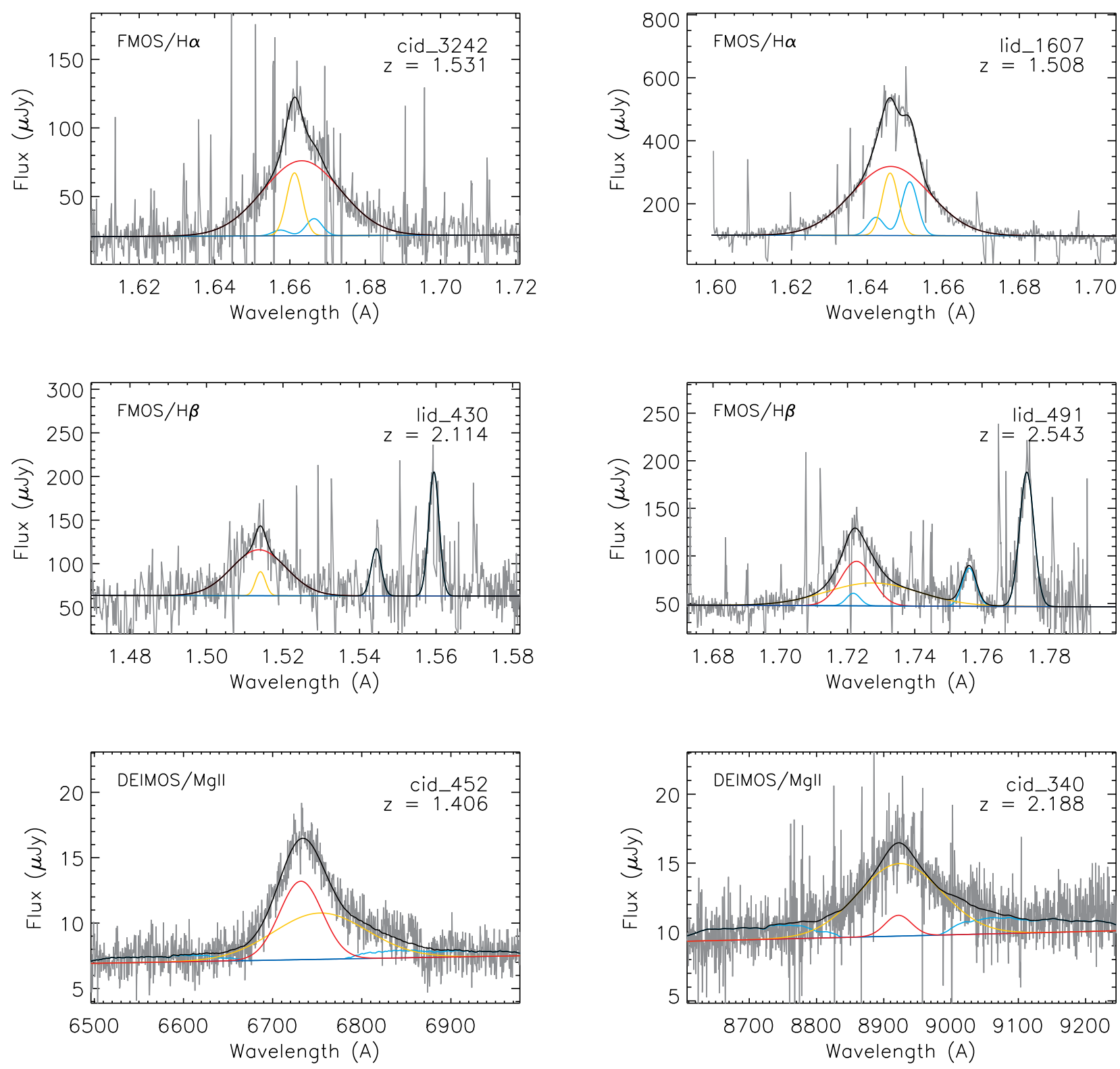

Figure 2. Examples of broad-line fits for $\mathrm{H} \alpha$ (top panels), $\mathrm{H} \beta$ (middle panels), and Mg II (bottom panels) emission lines for our sample of AGNs. The observed spectrum (gray) with the best-fit model (black) is indicated. The power-law continuum, narrow-line components (blue), Fe II emission component (blue), and broad-line components (red and yellow) are also indicated with colored curves.

we fit with one or two broad Gaussian components. Finally, we measure the broad-line width and the continuum/line luminosity from the best-fit spectra. The uncertainties of the continuum luminosity are calculated from the average of noise spectrum in the continuum wavelength range. Figure 2 shows examples of broad-line fits for the $\mathrm{H} \alpha$ (top), $\mathrm{H} \beta$ (middle), and Mg II (bottom) emission lines, respectively.

We compute $\mathrm{BH}$ masses using the equation in Schulze et al. (2018, $\mathrm{H} \alpha$ and $\mathrm{H} \beta$; provided by Vestergaard \& Peterson 2006) and Trakhtenbrot \& Netzer (2012, Mg II):

$$
M_{\mathrm{BH}}=10^{6.71}\left(\frac{L_{H \alpha}}{10^{42} \operatorname{ergs~s}^{-1}}\right)^{0.48}\left(\frac{\mathrm{FWHM}_{\mathrm{H} \alpha}}{10^{3} \mathrm{~km} \mathrm{~s}^{-1}}\right)^{2.12} \mathrm{M}_{\odot}
$$


Table 1. Broad-line AGN Sample

\begin{tabular}{|c|c|c|c|c|c|c|}
\hline Object ID & Redshift & $\begin{array}{c}\log M_{\mathrm{BH}} \\
\left(M_{\odot}\right)\end{array}$ & $\begin{array}{l}\log L_{\mathrm{bol}} \\
\left(\operatorname{erg~s}^{-1}\right)\end{array}$ & $\begin{array}{c}\log M_{\text {stellar }} \\
\left(M_{\odot}\right)\end{array}$ & Instrument & Line \\
\hline (1) & (2) & (3) & (4) & $(5)$ & (6) & (7) \\
\hline cid-36 & 1.826 & $9.38 \pm 0.06$ & 45.63 & $12.18_{-0.04}^{+0.00}$ & DEIMOS & Mg II \\
\hline cid-61 & 1.478 & $8.62 \pm 0.00$ & 45.38 & $11.48_{-0.15}^{+0.00}$ & DEIMOS & Mg II \\
\hline cid-66 & 1.512 & $8.45 \pm 0.03$ & 45.77 & $11.21_{-0.01}^{+0.24}$ & DEIMOS & Mg II \\
\hline cid-69 & 0.979 & $8.42 \pm 0.08$ & 45.68 & $11.22_{-0.06}^{+0.19}$ & DEIMOS & Mg II \\
\hline cid-70 & 1.638 & $8.85 \pm 0.11$ & 45.28 & $11.59_{-0.03}^{+0.16}$ & DEIMOS & Mg II \\
\hline cid-87 & 1.606 & $8.77 \pm 0.11$ & 46.69 & $11.61_{-0.00}^{+0.09}$ & DEIMOS & Mg II \\
\hline cid-98 & 1.506 & $7.74 \pm 0.05$ & 45.59 & $10.79_{-0.64}^{+0.40}$ & DEIMOS & Mg II \\
\hline cid-102 & 1.847 & $8.75 \pm 0.23$ & 45.81 & $11.09_{-0.28}^{+0.14}$ & DEIMOS & Mg II \\
\hline$\ldots$ & $\ldots$ & $\ldots$ & $\ldots$ & $\ldots$ & $\ldots$ & $\ldots$ \\
\hline
\end{tabular}

Note - Column (1) object ID (Marchesi et al. 2016). Column (2) redshift. Column (3) BH mass derived from virial method. Column (4) AGN bolometric luminosity. Column (5) total stellar mass derived from SED fitting (Suh et al. 2019). Column (6) instrument for spectroscopy. Column (7) broad emission line used for the BH mass measurement.

$M_{\mathrm{BH}}=10^{6.91}\left(\frac{L_{5100}}{10^{42} \operatorname{ergs~s}^{-1}}\right)^{0.50}\left(\frac{\mathrm{FWHM}_{\mathrm{H} \beta}}{10^{3} \mathrm{~km} \mathrm{~s}^{-1}}\right)^{2.0} \mathrm{M}_{\odot}$ slightly higher than, but consistent with independently calibrated by Grier et al. (2017, $M_{\mathrm{BH}}=10^{6.748}\left(\frac{L_{3000}}{10^{44} \mathrm{ergs} \mathrm{s}^{-1}}\right)^{0.62}\left(\frac{\mathrm{FWHM}_{\mathrm{MgII}}}{\mathrm{km} \mathrm{s}^{-1}}\right)^{2.0} \mathrm{M}_{\odot}$ $\left.f_{\text {vir }} \sim 3.5\right)$ via direct modeling of the BLR structure and dynamics. While the measurement uncertainties on $M_{\mathrm{BH}}$ are relatively small $(\sim 0.1$ where FWHM is the FWHM of the line in units of $1000 \mathrm{~km} \mathrm{~s}^{-1}$, and $L_{H \alpha}$ is the luminosity of broad $\mathrm{H} \alpha$ line. The $L_{5100}$ and $L_{3000}$ are the monochromatic continuum luminosities at rest-frame $5100 \AA$ and $3000 \AA$, respectively. The number of sources whose $M_{\mathrm{BH}}$ were derived using $\mathrm{H} \alpha, \mathrm{H} \beta$, and $\mathrm{Mg} \mathrm{II}$ lines are 21 (2 from DEIMOS, 19 from FMOS), 5 (2 from DEIMOS, 3 from FMOS), and 74 (all from DEIMOS), respectively.

The BH estimators we used are based on the mean virial coefficient $\epsilon \sim 1$ (e.g., Onken et al. 2004; Grier et al. 2013), corresponding to the mean virial factor of $f_{\text {vir }} \sim 4-5$, which is dex), the systematic uncertainties associated with different single-epoch virial calibrations carry a scatter of $\sim 0.3$ dex (e.g., McGill et al. 2008; Trakhtenbrot \& Netzer 2012; Shen 2013). We determine the uncertainties of $\mathrm{BH}$ mass given by the sum of the statistical and systematic uncertainties.

In Figure 3 we show AGN bolometric luminosity versus $\mathrm{BH}$ mass for our sample of broad-line AGNs in the different redshift bins. The AGN bolometric luminosity is derived using the absorption-corrected rest-frame 2$10 \mathrm{keV}$ luminosity by applying a luminosity- 


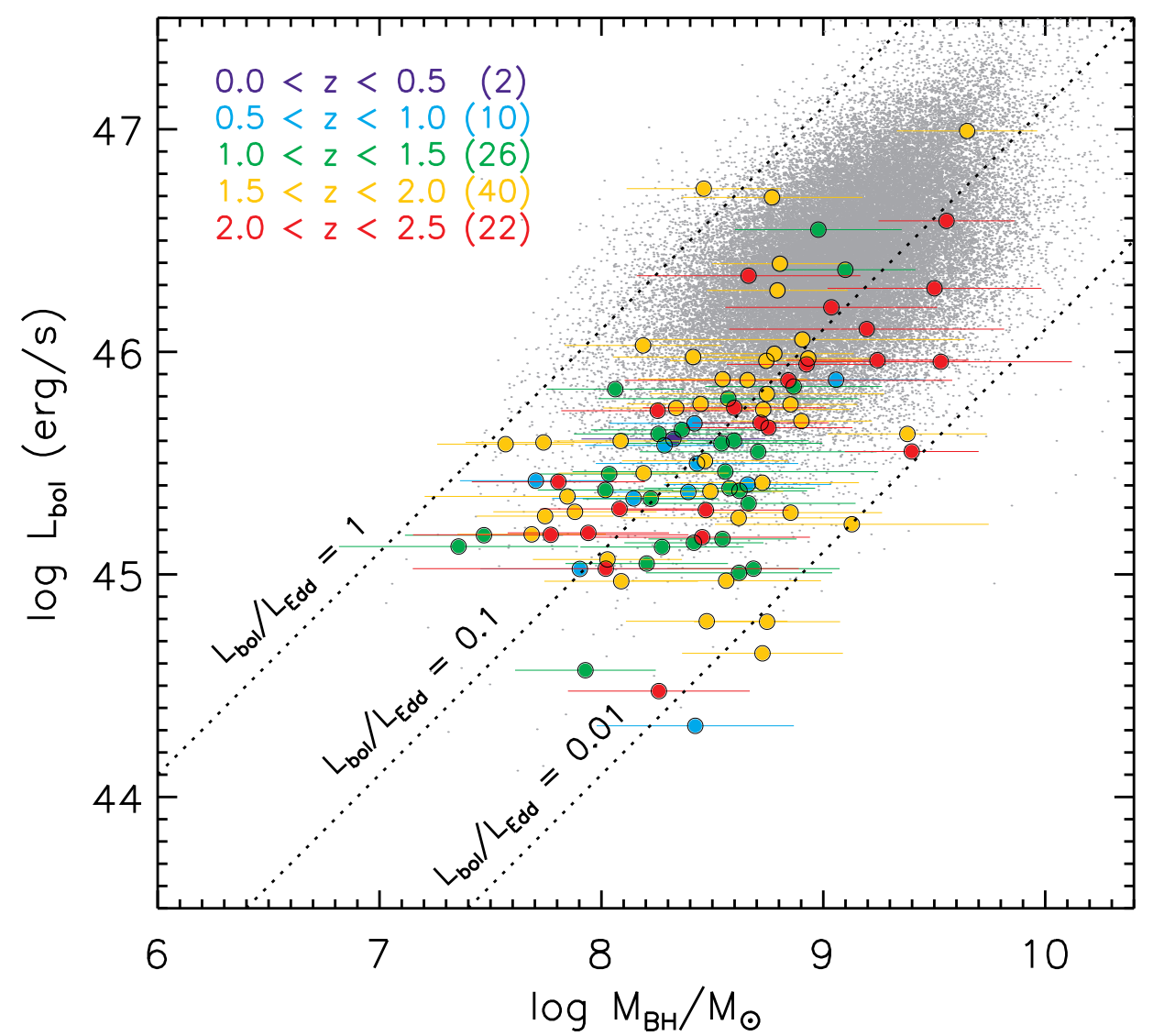

Figure 3. AGN bolometric luminosity versus black hole mass for our sample of broad-line AGNs in the different redshift bins. The numbers in parentheses refer to number of sources in each redshift bin. The SDSS quasar sample (gray points; Shen et al. 2011) is shown for comparison. As a reference, lines of constant Eddington ratio $\left(L_{\mathrm{bol}} / L_{\mathrm{Edd}}\right)$ equal to $1,0.1$, and 0.01 are plotted as dotted lines.

dependent bolometric correction described in Marconi et al. (2004, see Marchesi et al. 2016; Suh et al. 2019). For comparison, we show the SDSS DR7 quasar sample (gray points; Shen et al. 2011), which is limited to the highmass and high-luminosity regime due to the SDSS spectroscopic follow-up flux limit. Our sample of broad-line AGNs covers the $\mathrm{BH}$ mass range $7.0<\log \left(M_{\mathrm{BH}} / M_{\odot}\right)<9.5$ and the bolometric luminosity range $44<\log L_{\text {bol }} \operatorname{erg~s}^{-1}<$ 47 with the median value of Eddington ratios $L_{\text {bol }} / L_{\text {Edd }} \sim 0.1$. We list our sample of broad-line AGNs in Table 1, which includes BH masses, AGN bolometric luminosities, and host total stellar masses.

\section{THE $M_{\mathrm{BH}}-M_{\mathrm{STELLAR}}$ RELATION BEYOND THE LOCAL UNIVERSE}

We present the distribution of our sample of broad-line AGNs and their host galaxies at high redshift on the $M_{\mathrm{BH}}-M_{\text {stellar }}$ diagram in Figure 4. We also show the inactive sample of early-type galaxies at local universe and the local AGNs taken from Reines and Volonteri $(2015)^{1}$. Reines and Volonteri (2015) sample

\footnotetext{
${ }^{1}$ We correct the stellar masses of Reines and Volonteri (2015) sample to match the mass-dependent offset following the procedure outlined in Shankar et al. (2019). Reines and Volonteri (2015) adopt BH masses based on a mean virial factor of $f_{\text {vir }}=4.3$ from Grier et al. (2013), consistent with our value.
} 


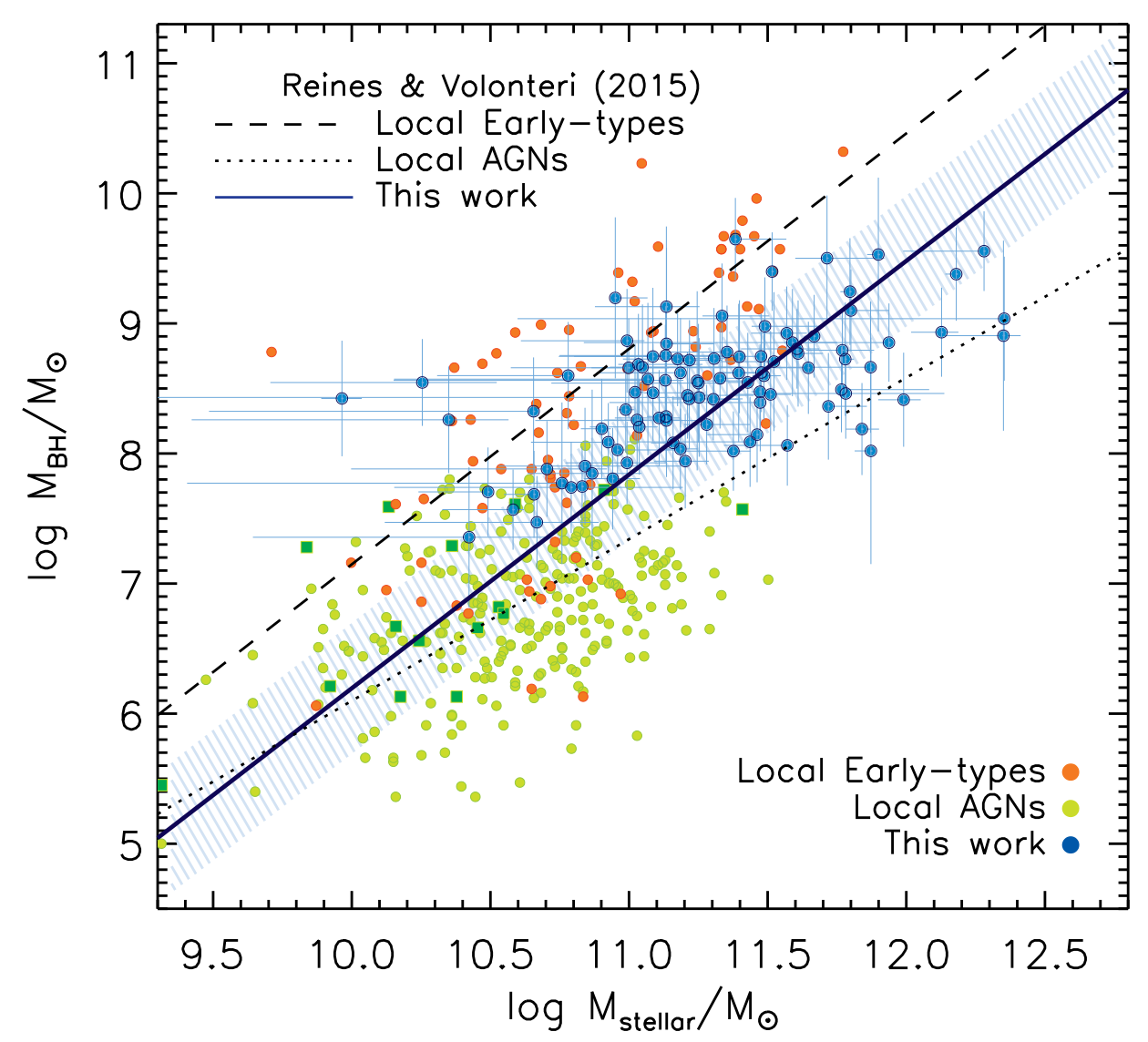

Figure 4. BH mass versus galaxy total stellar mass for our sample of 100 broad-line AGNs (blue circles). The orange circles indicate the inactive sample of elliptical galaxies and spiral/S0 galaxies with classical bulges at local universe (Reines and Volonteri 2015, taken from Kormendy \& Ho 2013), and the light green symbols indicate the local AGNs from Reines and Volonteri (2015). The 15 reverberation-mapped AGNs from Reines and Volonteri (2015, with BH masses taken from Bentz \& Katz 2015) are shown as dark green squares. The dashed and dotted lines indicate the local $M_{\mathrm{BH}}-M_{\text {stellar }}$ relation derived using total stellar mass from Reines and Volonteri (2015), as a reference. We show the $M_{\mathrm{BH}}-M_{\text {stellar }}$ relation for the combined sample of local inactive early-types, local AGNs, and our high-z AGNs as a blue solid line with the $1 \sigma$ scatter.

includes 262 broad-line AGNs based on the Sloan Digital Sky Survey (SDSS) Data Release 8 (DR8) spectroscopic catalog, and a subsample of 15 reverberation-mapped AGNs taken from Bentz \& Katz (2015), as well as 79 galaxies with dynamical $\mathrm{BH}$ mass taken from Kormendy \& Ho (2013).

We derive the linear correlation between $\mathrm{BH}$ mass and total stellar mass for the combined sample of local inactive early-types, local AGNs (Reines and Volonteri 2015), and our sample of high-redshift AGNs using a Bayesian approach to take into account uncertainties (Kelly 2007). We find the relation as

$$
\begin{aligned}
\log M_{\mathrm{BH}} / M_{\odot}= & (1.47 \pm 0.07) \times \log M_{\text {stellar }} / M_{\odot} \\
& -(8.56 \pm 0.04)
\end{aligned}
$$

with the intrinsic scatter of $\sim 0.5$ dex. We also derive the $M_{\mathrm{BH}}-M_{\text {stellar }}$ relation for our sample of high-redshift AGNs alone, and for the combined sample of local AGNs and our highredshift AGN sample. The derived linear relations are shown in the Figure 4 and 5. 

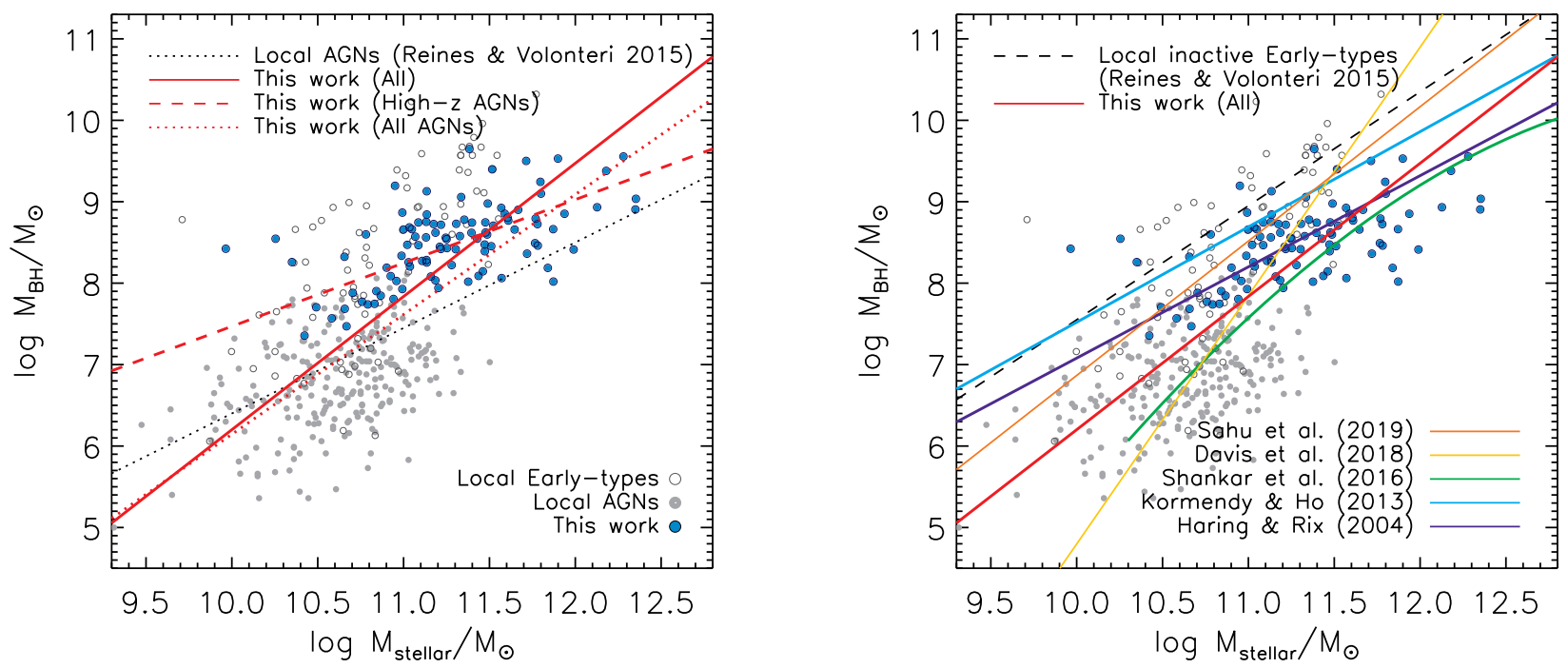

Figure 5. Same as Figure 4, but indicated with the local scaling relations for AGNs (left) and those for inactive galaxies hosting dynamically-detected BHs (right), for comparison. The red solid line indicates our derived $M_{\mathrm{BH}}-M_{\text {stellar }}$ relation for the combined sample of local inactive early-types (open circles), local AGNs (gray circles) from Reines and Volonteri (2015), together with our sample of high-z AGNs (blue circles). The $M_{\mathrm{BH}}-M_{\text {stellar }}$ relations only for our high-z AGNs and for all combined AGNs (local AGNs + our high-z AGNs) are indicated as a red dashed and dotted lines, respectively. The local $M_{\mathrm{BH}}-M_{\text {bulge }}$ relations from Häring \& Rix (2004, purple) and Kormendy \& Ho (2013, blue) are indicated. The local $M_{\mathrm{BH}}-M_{\text {stellar }}$ relations for early-type galaxies (Sahu et al. 2019, orange) and those for late-type galaxies (Davis et al. 2018, yellow) are also plotted. We also show the local intrinsic/unbiased $M_{\mathrm{BH}}-M_{\text {stellar }}$ relation predicted by Shankar et al. (2016) plotted as a green curve.

Our sample of AGN host galaxies at high redshift shows a modest offset from the local AGN relation (Reines and Volonteri 2015), while the mass range of our sample corresponds to that for the most massive early-type galaxies with inactive BHs in the local universe. It seems that AGN host galaxies beyond the local universe occupy, on average, a region between the relation of local AGNs and those of local earlytype galaxies with inactive BHs. We note that our sample of AGNs has a lower limit to the $\mathrm{BH}$ mass of $\log M_{\mathrm{BH}} \sim 7.04 M_{\odot}$ at $z=2$.

We compare our results with the local $M_{\mathrm{BH}}-$ $M_{\text {bulge }}$ relations in the right panel of Figure 5 (Häring \& Rix 2004; Kormendy \& Ho 2013). Our sample of AGNs tend to lie below the Kormendy \& Ho (2013) relation for local inactive early-type galaxies at a given $M_{\text {stellar }}$. It appears that the local $M_{\mathrm{BH}}-M_{\text {stellar }}$ relation of
Davis et al. (2018) is consistent with our data, even though only based on late-type galaxies, while the relation of Sahu et al. (2019), which is only based on early-type galaxies, seems to be inconsistent with our high-redshift AGNs. Shankar et al. (2016) proposed the local intrinsic relation by correcting the selection bias in the $M_{\mathrm{BH}}-M_{\text {stellar }}$ relation of inactive $\mathrm{BHs}$ with dynamically-measured masses. Indeed, we show that even our high-redshift sample of AGNs well matches the proposed local intrinsic $M_{\mathrm{BH}}-M_{\text {stellar }}$ relation within the scatter, further corroborating the presence of a bias in local inactive $\mathrm{BHs}$, and a negligible evolution in the intrinsic $M_{\mathrm{BH}}-M_{\text {stellar relation at least }}$ up to $z \sim 2.5$. Recently, Izumi et al. (2019) also found that the low-luminosity quasars discovered by Subaru High-z Exploration of Low-Luminosity Quasars (SHELLQs) survey 

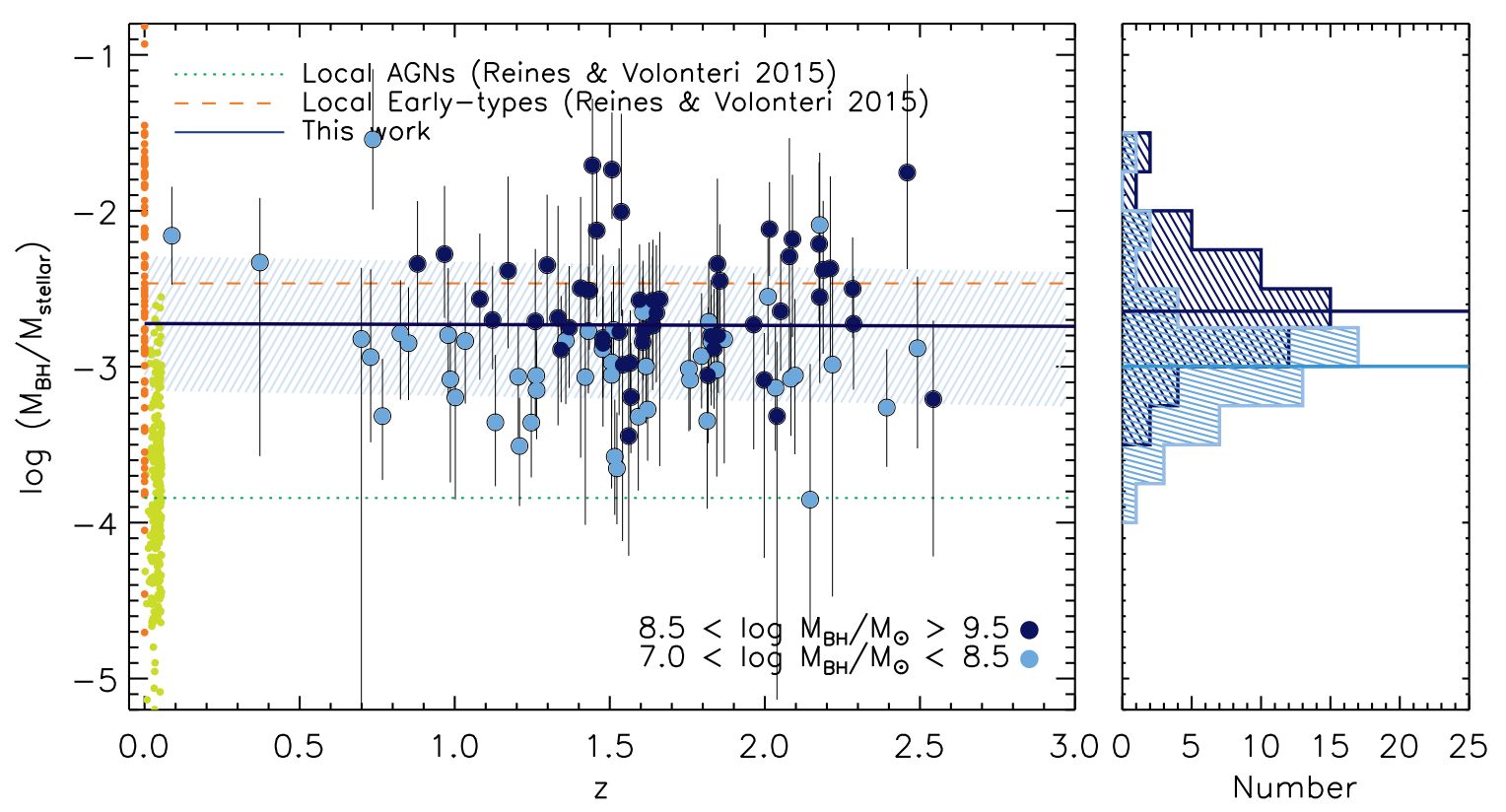

Figure 6. Evolution of $M_{\mathrm{BH}}$ to $M_{\text {stellar }}$ ratios. The green dotted and orange dashed lines represent the

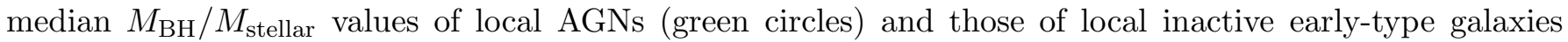
(orange circles) from Reines and Volonteri (2015), respectively. The solid line represents the best-fit trend for our sample of AGNs. We divided our sample into AGNs with less massive BHs $\left(7.0<\log M_{\mathrm{BH}} / M_{\odot}<8.5\right)$ and AGNs with massive BHs $\left(8.5<\log M_{\mathrm{BH}} / M_{\odot}<9.5\right)$.

appear to be located on or even below the local $M_{\mathrm{BH}}-M_{\text {bulge }}$ relation, even at $z \sim 6$.

In Figure 6, we show the histogram of $\mathrm{BH}-$ to-stellar mass ratios $\left(\log M_{\mathrm{BH}} / M_{\text {stellar }}\right)$ and the trend with redshift for our sample of AGN host galaxies in the two $\mathrm{BH}$ mass bins. We indicate the median $M_{\mathrm{BH}} / M_{\text {stellar values for the local }}$ AGNs and the local inactive early-type galaxies from Reines and Volonteri (2015) as a reference. Overall, we find no significant evolution of the $M_{\mathrm{BH}}-M_{\text {stellar }}$ relation with $M_{\mathrm{BH}} / M_{\text {stellar }} \sim$ $0.2 \%$ up to $z \sim 2.5$, which is markedly higher, by an order of magnitude, than local AGNs $\left(M_{\mathrm{BH}} / M_{\text {stellar }} \sim 0.015 \%\right)$, while smaller than the $\mathrm{BH}$-to-bulge mass ratios derived from inactive early-type galaxies $\left(M_{\mathrm{BH}} / M_{\text {bulge }} \sim 0.4 \%\right)$ in the local universe. We note that the $\mathrm{BH}$ mass range of local AGNs from Reines and Volonteri (2015) is $5.5<\log M_{\mathrm{BH}} / M_{\odot}<8.0$. It seems that galaxies with less massive $\mathrm{BHs}$ tend to have smaller $M_{\mathrm{BH}} / M_{\text {stellar }}$ ratios than those with more massive BHs. It is also interesting to note that the scatter does not depend much on redshift.

Our results are consistent with some previous studies (e.g., Shields et al. 2003; Salviander et al. 2007; Jahnke et al. 2009; Cisternas et al. 2011; Shen et al. 2008, 2015) of no evolution in $M_{\mathrm{BH}} / M_{\text {stellar }}$ ratios with redshift, while in contrast to other studies indicating that BHs predate the growth of their host galaxies (e.g., Treu et al. 2007; Shen et al. 2008; Woo et al. 2008; Merloni et al. 2010; Decarli et al. 2010; Bennert et al. 2011). We compare our findings with data from the zCOSMOS bright spectroscopic survey (Merloni et al. 2010), ${ }^{2}$ in the top panel of Figure 7. Compared to a sample of 89 Type 1 AGNs from Merloni et al. (2010), our

2 We corrected the stellar masses of Merloni et al. (2010) by subtracting $\sim 0.25$ dex, to take into account for the different IMF. 


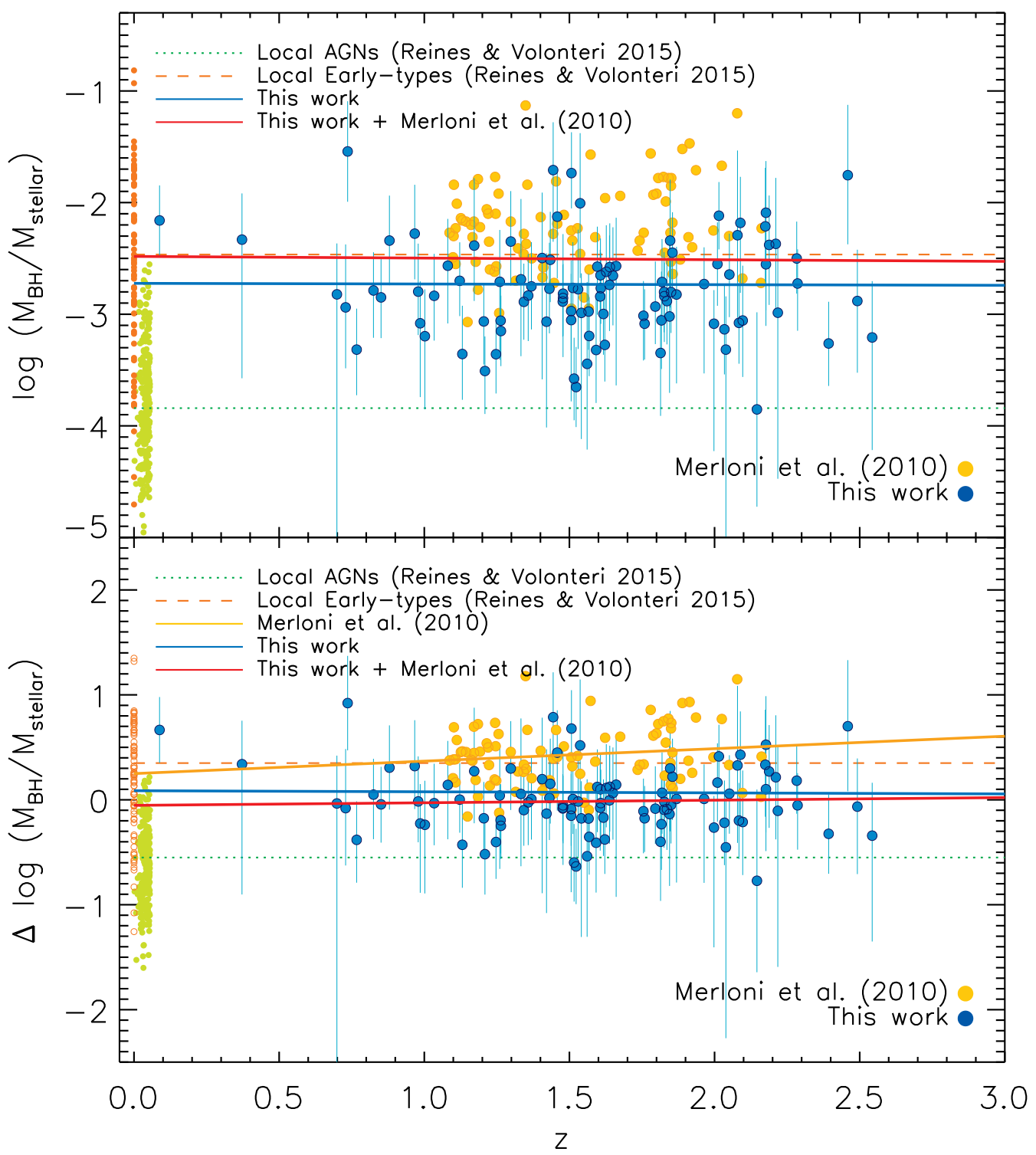

Figure 7. Top: redshift evolution of $M_{\mathrm{BH}}$-to- $M_{\text {stellar }}$ ratios for our sample of AGNs (blue) in comparison with data from the literature, plotted as yellow symbols (Merloni et al. 2010). The orange and the green circles indicate the individual local inactive early-type galaxies and the local AGNs from Reines and Volonteri (2015). Blue line indicates the best-fit trend for our sample of AGNs (see Figure 6). Red line shows the best-fit of combined sample of our AGNs (blue) with the data from Merloni et al. (2010, yellow). The dotted and dashed lines represent the median values of local AGNs (green circles) and those of local inactive early-type galaxies (orange circles) from Reines and Volonteri (2015), respectively. Bottom: evolution of the offset ( $\Delta \log M_{\mathrm{BH}} / M_{\text {stellar }}$ ) from the local relation of Häring \& Rix (2004), which is calculated as the distance of each point to the Häring \& Rix (2004) correlation. Yellow line shows the best-fit of Merloni et al. (2010), and blue line shows the best-fit of our sample of AGNs. Red line indicates the best-fit trend for the combination of our sample of AGNs and those of Merloni et al. (2010). 
sample of AGNs, which are selected by deep Chandra X-ray, were able to fill in the lower BH mass region by reaching to lower luminosities with the deeper spectroscopy that were missed in earlier luminous sample at high redshift.

We further explore the deviation of our sample of AGN host galaxies from the local scaling relation by measuring the offset, $\Delta \log M_{\mathrm{BH}} / M_{\text {stellar}}$, perpendicular to the Häring \& Rix (2004) relation, following a same approach as Merloni et al. (2010). We note that the local sample of Häring \& Rix (2004) is mostly bulge-dominated (i.e., $M_{\text {bulge }} \sim M_{\text {stellar }}$ ). The bottom panel of Figure 7 shows the $\log M_{\mathrm{BH}} / M_{\text {stellar }}$ relative to the local relation of Häring \& Rix (2004) as a function of redshift. We show the zCOSMOS AGN sample from Merloni et al. (2010) with their best-fit relation: $\Delta \log \left(M_{\mathrm{BH}} / M_{\text {stellar }}\right)=0.68 \log (1+z)$. We conclude that we do not find a significant cosmic evolution of the $M_{\mathrm{BH}} / M_{\text {stellar }}$ ratio that our sample of moderate-luminosity AGNs, together with the relatively bright AGN sample of Merloni et al. (2010) are indeed broadly consistent with the local $M_{\mathrm{BH}}-$ $M_{\text {bulge }}$ relation (Häring \& Rix 2004) up to $z \sim 2.5$, in agreement with no evolution of the scaling relation suggested by previous studies (e.g., Shields et al. 2003; Salviander et al. 2007; Shen et al. 2008; Cisternas et al. 2011; Shen et al. 2015).

While there could be potential systematics and selection biases due to incompleteness in stellar mass and $\mathrm{BH}$ mass measurements in the resulting $M_{\mathrm{BH}}-M_{\text {stellar }}$ relation (i.e., Lauer et al. 2007; Shen et al. 2015; Shankar et al. 2016, 2019), i.e., a threshold in luminosity and the detectability of the broad emission line, on average, could lead a bias toward more massive $\mathrm{BHs}$ with respect to the galaxy stellar mass, we probe sufficiently lowluminosity AGNs at high redshift, covering the population of less massive BHs.

\section{DISCUSSION}

In this work we provide evidence for a nonevolving $M_{\mathrm{BH}}-M_{\text {stellar }}$ relation. This single observation has profound implications on several aspects of galaxy evolution. Direct time integration of the total emissivity of AGN, suggests a decrease of the $\mathrm{BH}$ mass density of a factor of $\sim 3$ up to $z \sim 2$, assuming a constant radiative efficiency (see e.g., bottom panels of Figure 4 in Shankar et al. 2013). A constant $M_{\mathrm{BH}} / M_{\text {stellar }}$ ratio would then imply a proportionally similar evolution in the integrated stellar mass density of galaxies, which might indeed be the case (e.g., Figure 27 in Bernardi et al. 2010), though systematics in stellar mass measurements still prevent a robust conclusion in this respect (e.g., Bernardi et al. 2017). This result of a constancy in the $M_{\mathrm{BH}}-M_{\text {stellar }}$ relation is also in line with other independent lines of evidence. For example, several studies suggested a weak evolution in the $M_{\mathrm{BH}}-\sigma$ relation, either based on Soltan-type arguments or direct observations (e.g., Gaskell \& Kormendy 2009; Shankar et al. 2009; Zhang et al. 2012; Shen et al. 2015).

A non-evolving $M_{\mathrm{BH}}-\sigma$ and $M_{\mathrm{BH}}-M_{\text {stellar }}$ relation would have significant implications on the overall co-evolution of the BHs and galaxies and on the fundamental plane of early-type galaxies. This would necessarily also imply a weak evolution in the relation of the $\sigma-M_{\text {stellar }}$ at least in early-type, bulge-dominant galaxies. If in the central regions one can approximate $M_{\mathrm{BH}} \sim M_{\text {stellar }} \sim M_{\mathrm{dyn}} \sim k \times R_{e} / \sigma^{2}$, at fixed $\mathrm{BH}$ mass and velocity dispersion one would naively expect a constant product $k \times R_{e}$. If early-type galaxies decrease in size $R_{e}$ at higher redshifts (e.g., van Dokkum et al. 2013), this would imply a proportional increase in the virial constant $k$, which is expected if the Sérsic index decreases at earlier epochs (e.g., Figure 1 in Bernardi et al. 2018), which is expected in merger-dominated models (e.g., Hilz et al. 2013). 
We suggest that the $\mathrm{BHs}$ and galaxies both have grown predominantly at higher redshift $(z>3)$, and all necessary stellar mass may already exist in galaxies at $z \sim 3$. This is in line with the fact that their growth rates seem to be broadly correlated, both in the integrated sense, i.e., SFR density and $\mathrm{BH}$ accretion rate density, and the individual sense, i.e., SFR and $L_{\mathrm{AGN}}$ (e.g., Shankar et al. 2013; Suh et al. 2019). In the later stage of AGN evolution, the moderateluminosity AGNs with relatively high accreting $\mathrm{BHs}$ found below the local scaling relation will presumably move up to the $M_{\mathrm{BH}}-M_{\text {stellar }}$ plane induced by secular processes, becoming eventually inactive galaxies harboring SMBHs. The secular process may not change the overall $M_{\mathrm{BH}}-M_{\text {stellar }}$ ratio by feeding both $\mathrm{BH}$ and stellar masses.

We note here that there could be an evolution in the $M_{\mathrm{BH}}-M_{\text {bulge }}$ relation, considering that our sample of AGNs are not purely bulgedominated (see e.g., Jahnke et al. 2009). To explain the tight relationship between the final $\mathrm{BH}$ and spheroid bulge mass, a re-distribution of stellar masses is required from the disk to the bulge, also induced by secular processes such as disk instabilities. Elbaz et al. (2018) found that the galaxies hosting an AGN at $z \sim 2$ appear to be systematically associated with the most compact star-forming galaxies, suggesting that the physical mechanism responsible for the rise in star-formation compactness also efficiently feeds the central BH, or possibly that the AGN activity plays a role in triggering the compact starformation through positive feedback, i.e., bulgeformation (see also Chang et al. 2017). These results suggest that the majority of AGN host galaxies at $z<3$ might be driven more by internal secular processes, implying that they have substantially grown at a much earlier epoch.

\section{CONCLUSIONS}

We have studied the evolution of relations between BH mass and total stellar mass for 100 X-ray-selected moderate-luminosity, broad-line AGNs in the CCLS up to $z \sim 2.5$. By taking advantage of the deep multi-wavelength photometry and the unique, intensive spectroscopy in the COSMOS field, we measured in a uniform way the galaxy total stellar mass using a SED decomposition technique and the $\mathrm{BH}$ masses based on single-epoch virial estimates. The main results are summarized as follows.

- Our sample of AGN host galaxies has total stellar masses in the range $10^{10-12} M_{\odot}$, and the $\mathrm{BH}$ masses are broadly distributed around $10^{7.0-9.5} M_{\odot}$, probing sufficiently low-luminosity AGNs at high redshift covering the less massive $\mathrm{BH}$ populations. The median Eddington ratios is $L_{\mathrm{bol}} / L_{\mathrm{Edd}} \sim 0.1$.

- Our sample of AGN host galaxies beyond the local universe tend to lie below the $M_{\mathrm{BH}}-M_{\text {stellar }}$ relations for local inactive early-type galaxies at a given $M_{\text {stellar }}$, with no evident evolution with redshift.

- By combining our moderate-luminosity AGNs with the relatively bright zCOSMOS AGN sample (Merloni et al. 2010), we find that the distribution of $M_{\mathrm{BH}}-$ $M_{\text {stellar }}$ relations for high-redshift AGNs is broadly consistent with the local value within uncertainties, with $M_{\mathrm{BH}} / M_{\text {stellar }} \sim$ $0.3 \%$ up to $z \sim 2.5$.

We thank the anonymous referee for valuable comments that significantly improved this work. FS acknowledges partial support from a Leverhulme Trust Research Fellowship. VA acknowledges funding from the European Union's Horizon 2020 research and innovation programme under grant agreement No749348. 


\section{REFERENCES}

Aird, J., Coil, A. L., Georgakakis, A., et al. 2015, MNRAS, 451, 1892

Bennert, V. N., Auger, M. W., Treu, T., Woo, J.-H., \& Malkan, M. A. 2011, ApJ, 742, 107

Bentz, M. C., \& Katz, S. 2015, PASP, 127, 67

Bernardi, M., Sheth, R. K., Tundo, E., Hyde, J. B. 2007, ApJ, 660, 267

Bernardi, M., Shankar, F., Hyde, J. B., et al. 2010, MNRAS, 404, 2087

Bernardi, M., Meert, A., Sheth, R. K., et al. 2017, MNRAS, 468, 2569

Bernardi, M., Sheth, R. K., Fischer, J.-L., et al. 2018, MNRAS, 475, 757

Bongiorno, A., Maiolino, R., Brusa, M., et al. 2014, MNRAS, 443, 2077

Bruzual, G., \& Charlot, S. 2003, MNRAS, 344, 1000

Calzetti, D., Armus, L., Bohlin, R. C., et al. 2000, ApJ, 533, 682

Caplar, N., Lilly, S. J., Trakhtenbrot, B. 2015, ApJ, 811, 148

Caplar, N., Lilly, S. J., Trakhtenbrot, B. 2018, ApJ, 867, 148

Cisternas, M., Jahnke, K., Bongiorno, A., et al. 2011, ApJL, 741, L11

Civano, F., Marchesi, S., Comastri, A., et al. 2016, ApJ, 819, 62

Chabrier, G. 2003, ApJ, 586L, 133

Chang, Y.-Y., Le Floc'h, E., Juneau, S., et al. 2017, MNRAS, 466, L103

Chary, R., \& Elbaz, D. 2001, ApJ, 556, 562

Dale, D. A., \& Helou, G. 2002, ApJ, 576, 159

Davis, B. L., Graham, A. W., Cameron, E. 2018, ApJ, 869, 113

Decarli, R., Falomo, R., Treves, A., et al. 2010, MNRAS, 402, 2453

Di Matteo, T., Springel, V., \& Hernquist, L. 2005, Nature, 433, 604

Elbaz, D., Leiton, R., Nagar, N., et al. 2018, A\&A, 616, A110

Faber, S. M., Phillips, A. C., Kibrick, R. I., et al. 2003, Proc. SPIE, 4841, 1657

Ferrarese, L., \& Merritt, D. 2000, ApJL, 539, L9

Gaskell, C. M. \& Kormendy, J. 2009, ASPC, 419, 388

Gebhardt, K., Bender, R., Bower, G., et al. 2000, ApJL, 539, L13

Greene, J. E., \& Ho, L. C. 2005, ApJ, 630, 122
Greene, J. E., \& Ho, L. C. 2006, ApJ, 641, 117

Grier, C. J., Martini, P., Peterson, B. M., et al. 2013, ApJ, 773, 90

Grier, C. J., Pancoast A., Barth A. J. et al. 2017, ApJ, 849, 146

Gültekin, K., Richstone, D. O., Gebhardt, K., et al. 2009, ApJ, 698, 198

Harris, K. A., Williger, G. M., Haberzettl, L., et al. 2013, MNRAS, 435, 3125

Hasinger, G., Capak, P., Salvato, M., et al. 2018, ApJ, 858, 77

Häring N., \& Rix, H.-W. 2004, ApJL, 604, L89

Hils, M., Naab, T., Ostriker, J. P. 2013, MNRAS, 429, 2924

Hopkins, P. F., Somerville, R. S., Hernquist, L., et al. 2006, ApJ, 652, 864

Izumi, T., Onoue, M., Matsuoka, Y., et al. 2019, PASJ, accepted

Jahnke, K., Bongiorno, A., Brusa, M., et al. 2009, ApJL, 706, L215

Kashino, D., Silverman, J. D., Rodighiero, G., et al. 2013, ApJL, 777, L8

Kaspi, S., Smith, P. S., Netzer, H., et al. 2000, ApJ, 533, 631

Kelly, B. C. 2007, ApJ, 665, 1489

Kimura, M., et al. 2010, PASJ, 62, 1135

Kollmeier, J. A., Onken, C. A., Kochanek, C. S., et al. 2006, ApJ, 648, 128

Kormendy, J. \& Ho, Luis, C. 2013, ARA\&A, 51, 511

Laigle, C., McCracken, H. J., Ilbert, O., et al. 2016, ApJS, 224, 24

Lauer, T. R., Tremaine, S., Richstone, D. \& Faber, S. M. 2007, ApJ, 670, 249

Lilly, S. J., Le Fèvre, O., Renzini, A., et al. 2007, ApJS, 172, 70

Magorrian, J., Tremaine, S., Richstone, D., et al. 1998, AJ, 115, 2285

Marchesi, S., Civano, F., Elvis, M., et al. 2016, ApJ, 817, 34

Marconi, A., \& Hunt, L. K. 2003, ApJ, 589, L21

Marconi, A., Risaliti, G., Gilli, R., et al. 2004, MNRAS, 351, 169

Matsuoka, Y., Oyabu, S., Tsuzuki, Y., \& Kawara, K. 2007, ApJ, 663, 781

McConnell, N. J., \& Ma, C.-P. 2013, ApJ, 764, 184

McGill, K. L., Woo, J., Treu, T., \& Malkan, M. A. 2008, ApJ, 673, 703 
McLure, R. J., \& Dunlop, J. S. 2004, MNRAS, 352,1390

McLure, R. J, \& Jarvis, M. J. 2002, MNRAS, 337, 109

Merloni, A., Bongiorno, A., Bolzonella, M., et al. 2010, ApJ, 708, 137

Mejía-Restrepo, J. E., Trakhtenbrot, B., Lira, P., et al. 2016, MNRAS, 460, 187

Onken, C. A., Ferrarese, L., Merritti, D., et al. 2004, ApJ, 615, 645

Park, D., Woo, J.-H., Bennert, V. N., et al. 2015, ApJ, 799, 164

Peng, C. Y., Impey, C. D., Rix, H.-W., et al. 2006, ApJ, 649, 616

Prevot, M. L., Lequeux, J., Maurice, E., Prevot, L., Rocca-Volmerange, B. 1984, A\&A, 132, 389

Reines, A. E. and Volonteri, M. 2015, ApJ, 813, 82

Richards, G. T., Lacy, M., Storrie-Lombardi, L. J., et al. 2006, ApJS, 166, 470

Ross, N. P., McGreer, I. D., White, M., et al. 2013, ApJ, 773, 14

Sahu, N., Graham, A. W., Davis, B. L. 2019, ApJ, 876,155

Salviander, S., Shields, G. A., Gebhardt, K., \& Bonning, E. W. 2007, ApJ, 662, 131

Salviander, S., Shields, G. A. \& Bonning, E. W. 2015, ApJ, 799, 173

Sani, E., Marconi, A., Hunt, L. K., and Risaliti, G. 2011, MNRAS, 413, 1479

Schramm, M. \& Silverman, J. D. 2013, ApJ, 767, 13

Schulze, A. \& Wisotzki, L. 2014, MNRAS, 438, 3422

Schulze, A., Silverman, J. D., Kashino, D., et al. 2018, ApJS, 239, 22

Shankar, F., Bernardi, M., Haiman, Z. 2009, ApJ, 694,867

Shankar, F., Weinberg, D. H., Miralda-Escudé J. 2013, MNRAS, 428, 421

Shankar, F., Bernardi, M., Sheth, R. K., et al. 2016, MNRAS, 460, 3119

Shankar, F., Bernardi, M., Sheth, R. K. 2017, MNRAS, 466, 4029
Shankar, F., Bernardi, M., Richardson, K., et al. 2019, MNRAS, 485, 1278

Shen, Y. 2013, BASI, 41, 61

Shen, Y., Greene, J. E., Strauss, M. A., Richards, G. T., \& Schneider, D. P. 2008, ApJ, 680, 169

Shen, Y., Richards, G. T., Strauss, M. A., et al. 2011, ApJS, 194, 45

Shen, Y., Greene, J. E., Ho, L. C., et al. 2015, ApJ, 805, 96

Shields, G. A., Gebhardt, K., Salviander, S., et al. 2003, ApJ, 583, 124

Silk, J., \& Rees, M. J. 1998, A\&A, 331, L1

Silva, L., Maiolino, R., \& Granato, G. L. 2004, MNRAS, 355, 973

Silverman, J. D., Kashino, D., Sanders, D., et al. 2015, ApJS, 220, 12

Suh, H., Civano, F., Hasinger, G., et al. 2015, ApJ, 815, 129

Suh, H., Civano, F., Hasinger, G., et al. 2017, ApJ, 841, 102

Suh, H., Civano, F., Hasinger, G., et al. 2019, ApJ, 872, 168

Sun, M., Trump, J. R., Brandt, W. N., et al. 2015, ApJ, 802, 14

Trakhtenbrot, B., \& Netzer, H. 2010, MNRAS, 406, L35

Trakhtenbrot, B., \& Netzer, H. 2012, MNRAS, 427, 3081

Trakhtenbrot, B., Urry, C. M., Civano, F., et al. 2015, SCI, 349, 6244

Treu T., Woo J.-H., Malkan M. A., Blandford R. D. 2007, ApJ, 667, 117

van Dokkum, P. G., Leja, J., Nelson, E. J., et al. 2013, ApJ, 771, 35

Vestergaard, M., \& Wilkes, B. J. 2001, ApJS, 134, 1

Vestergaard, M. 2002, ApJ, 571, 733

Vestergaard, M., \& Peterson, B. M. 2006, ApJ, 641,689

Woo, J.-H., \& Urry, C. M. 2002, ApJ, 579, 530

Woo, J.-H., Treu T., Malkan M. A., Blandford R. D. 2008, ApJ, 681, 925

Zhang, X., Lu, Y., Yu, Q. 2012, ApJ, 761, 5 